\title{
Reduction of Neurogenesis with Social Isolation Decreases Pain Sensitivity in Tail Flick Test in Male Rats
}

\author{
Hamidreza Famitafreshi1, Morteza Karimian ${ }^{2 *}$ \\ ${ }^{1}$ Department of Physiology, Tehran University of Medical Science-International Campus, Tehran, Iran \\ ${ }^{2}$ Department of Physiology, Tehran University of Medical Science, Tehran, Iran \\ Email: *karimian@tums.ac.ir
}

How to cite this paper: Famitafreshi, $\mathrm{H}$. and Karimian, M. (2017) Reduction of Neurogenesis with Social Isolation Decreases Pain Sensitivity in Tail Flick Test in Male Rats. World Journal of Neuroscience, 7, 223-231

https://doi.org/10.4236/wjns.2017.72018

Received: April 7, 2017

Accepted: May 20, 2017

Published: May 23, 2017

Copyright $\odot 2017$ by authors and Scientific Research Publishing Inc. This work is licensed under the Creative Commons Attribution International License (CC BY 4.0).

http://creativecommons.org/licenses/by/4.0/

\begin{abstract}
Introduction: Pain is a complex phenomenon and in many diseases is the cardinal manifestation. In many of them, the source of pain is obscure and in turn curing pain also becomes difficult. Finding a new regulatory mechanism for pain perception and processing such as alternation of neurogenesis may establish a new treatment. Methods and Materials: In this study, 32 male Sprague-Dawley rats were randomly divided into four groups: social, isolated, morphine-treated socialized (MTS) and morphine-treated isolated (MTI). After injection of BrdU for 14 days $(50 \mathrm{mg} / \mathrm{kg} / \mathrm{rat} /$ day $/ \mathrm{i.p}$ ) and morphine for seven days from day 8 ( $3 \mathrm{mg} / \mathrm{kg} / \mathrm{rat} / \mathrm{day} / \mathrm{i} . \mathrm{p})$, rats were performed tail flick test and then sacrificed. Brains were prepared for assessing neurogenesis and serums were collected for assessing glutathione. Results: In tail flick test isolated and morphine-treated isolated rats had decreased sensitivity to pain stimuli compared to social and morphine-treated socialized rats, respectively. In assessing neurogenesis, isolated and morphinetreated isolated rats had reduced numbers of newly generated neurons compared to social and morphine-treated socialized rats, respectively. Glutathione in serum in isolated and morphine-treated isolated rats increased compared to social and morphine-treated socialized rats, respectively. Conclusion: Reduction of neurogenesis was associated with reduced pain sensitivity in isolated groups. So, isolation may alleviate pain and reduce pain threshold and sensitivity.
\end{abstract}

\section{Keywords}

Neurogenesis, Pain, Morphine, Glutathione, Social Isolation, Social and Tail Flick Test 


\section{Introduction}

Pain is a complex phenomenon that many scientists have been tried to describe it in an understandable way. Since the nature of pain in many situations is unknown, so pain cannot be described by only one mechanism by authorities. Therefore, there have been many investigations into nature and medications that can alleviate pain.

It is well known that reward system regulates reinforcing or diminishing behaviors. Reward circuit consists of hippocampus, amygdala and nucleus accumbens [1]. Pain as an unacceptable behavior is anticipated to reduce as the reward center does not support this behavior. Reward circuit efficacy seems positively regulated by a number of neurons that are generated in the hippocampus [2].

Production of new neurons in the brain after birth is called neurogenesis. Production of new neurons mainly occurs in two brain regions dentate gyrus of hippocampus and subventricular zone [3]. Since the hippocampus is part of reward circuit it is thought that changes in production of new neurons will affect the function of this important circuit.

The environment is one of the main factors that are thought to change the response to stimuli [4]. Environmental modulation can be modeled in animal studies by several models. In this study for modeling environmental change isolation and socialization have been used. According to previous studies, social isolation reduces brain cognitive function [5], and socialization improves cognitive functions [6]. In previous studies, changes in brain function along with alternation of neurogenesis in appositive manner have been investigated.

Dealing with pain especially intractable pain is one of the issues that have attained many efforts. In this study, it is investigated that if environmental modalities can change sensitivity to pain stimuli and also investigating the role of neurogenesis in this regard.

\section{Methods and Materials}

\subsection{Animal Care}

Thirty-two male Sprague-Dawley rats 8 to 10 weeks (200 - 250 grams) were housed in standard small $(27 \times 15 \times 21)$ or large $(42 \times 15 \times 21)$ polycarbonate cage with ad labium access to food and water. In controlled condition, cages were maintained in a temperature-controlled $\left(22^{\circ} \mathrm{C} \pm 2^{\circ} \mathrm{C}\right)$ and humidity-controlled $(40 \%-70 \%)$ vivarium with a $12 \mathrm{~h}$ light/dark cycle (lights on at 7:00 A.M.). All behavioral testing took place during the lights on the phase of the cycle (specifically, from 11:00 A.M. to 3:00 P.M.) in the experimental room. All rats were habituated to the Tehran University of Medical Science vivarium for at least $7 \mathrm{~d}$ before the start of experiments. All experimental protocols were in accordance with the Animal Ethics Committee of Tehran University of Medical sciences. In each group, 8 rats were used. In each group, 8 rats were used for inducing pair state (socializing). 


\subsection{Isolation}

Isolation of rats was done in small cages with black plastic cover $(27 \times 15 \times 21)$. During isolation, rats had free accessed to food and water. The light-dark cycle was not disturbed [7].

\subsection{Socialization}

Socialization of rats was done in large cages $(45 \times 15 \times 21)$. During pairing, rats had free accessed to food and water. The light-dark cycle was not disturbed [8].

\subsection{Morphine Treatment}

Morphine was first prepared from in powder form (Temad co.). Then it was diluted with distilled water. For inducing morphine tolerance, morphine was injected for 7 days from day 8 (3 mg/kg/day/i.p) [9].

\subsection{Tail Flick Experiment}

Tail-Flick Test is a nociceptive essay based on the measurement of the latency of the avoidance response to thermal stimulus in rodents. Basically, a thermal stimulus is applied to the tail; when the animal feels discomfort, it reacts by a sudden tail movement. The tail flick reaction time is then measured and used as an index of animal pain sensitivity [10].

\subsection{Neurogenesis}

For assessing neurogenesis each rat received BrdU (5-bromo-2'-deoxyuridine, Sigma-Aldrich Co) (50 mg/rat/day/i.p) for 14 days. After performing behavioral tests rats were anesthetized with ketamine $(100 \mathrm{mg} / \mathrm{kg})$. Rats' brains were fixed with paraformaldehyde $4 \%$. Histologic sections were prepared from hippocampus area of rat's brain. BrdU-positive neuron staining was done with anti-BrdU staining kit (Roche). BrdU-positive neurons were colored brown as single or cluster. BrdU-positive neuron counting was done by Image J software using a light microscope (Zeiss Co). Briefly, counting was done rostrocaudally and bilaterally. Total counts were multiplied by 5 [11].

\subsection{Glutathione Assay in Serum}

DTNB was used as the reaction substrate for estimating the amount of reduced glutathione. For performing this experiment Tris buffer, DTNB and methanol were used. 100 microliter of serum sample was added to the above mixture. The final solution developed yellow color, and maximum absorbance was measured at $412 \mathrm{~nm}$ with the spectrometer [12].

\subsection{Statistics}

Data were analyzed using SPSS version 22 and Graph pad prism. Univariate ANOVA with two factors (morphine-treated $\times$ socialization) was done for equality of variance and if the inequality was significant, post hoc test of Tukey was done for assessing of equality of means. Data were as represented as mean \pm 
SEM and $P<0.05$ considered significant. ${ }^{*}$ was used for showing a significant difference between groups that are beside together (social $\times$ isolation and morphine-treated socialized $($ MTS $) \times$ morphine-treated isolated $($ MTI)).

\section{Results}

\subsection{Tail Flick Test}

Latency to withdraw tail: In tail flick test isolated and morphine-treated isolated (MTI) rats had decreased sensitivity to pain stimuli compared to social and morphine-treated socialized (MTS) rats, respectively. It should be noted that after 7 days tolerance to morphine will occur (Figure 1).

\subsection{Neurogenesis}

Numbers of newly proliferated neurons: In assessing neurogenesis, isolated and morphine-treated isolated (MTI) rats had reduced numbers of newly generated neurons compared to social and morphine-treated socialized (MTS) rats, respectively (Figure 2 and Figure 3 ).

\subsection{Glutathione}

Serumic glutathione assessment: Glutathione in serum in isolated and morphine-treated isolated (MTI) rats increased compared to social and morphinetreated socialized (MTS) rats, respectively (Figure 4).

\section{Discussion}

In this study two results of great importance to be mentioned 1) socialization increases sensitivity to pain 2) reduction of neurogenesis reduces sensitivity to pain.

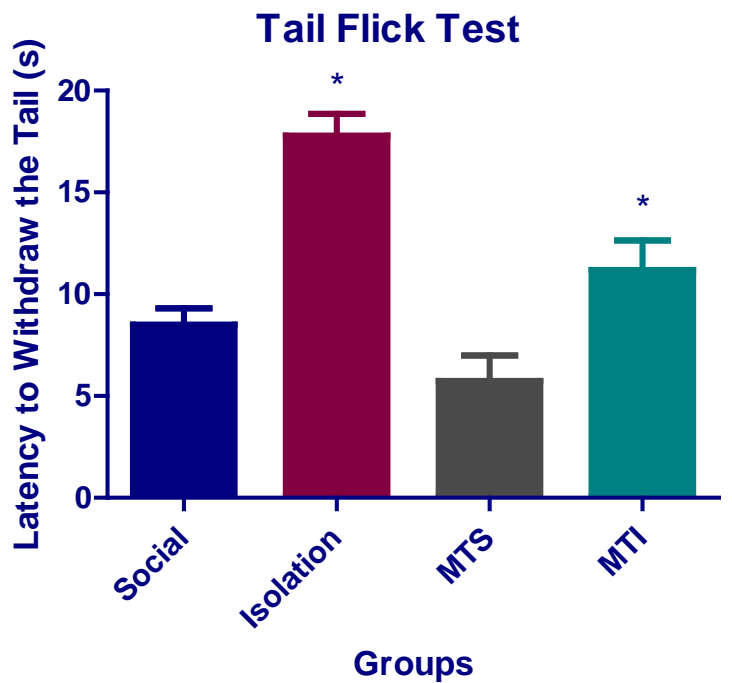

Figure 1. This figure illustrated the performance of rats in tail flick test $(n=8)$. Data were as represented as mean \pm SEM and $P<0.05$ considered significant. ${ }^{*}$ was used for showing a significant difference between groups that are beside together (social $\times$ isolation and morphine-treated socialized $(\mathrm{MTS}) \times$ morphine-treated isolated $(\mathrm{MTI})$ ). 


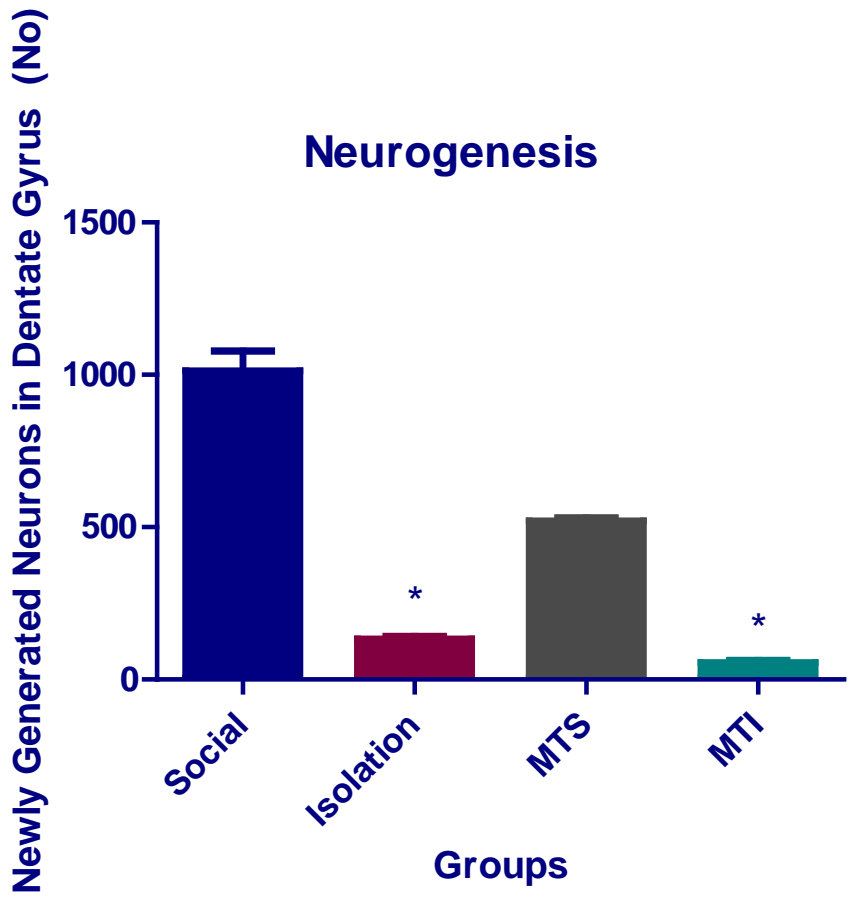

Figure 2. This figure illustrated number of newly proliferated neurons in the dentate gyrus of the hippocampus $(\mathrm{n}=8)$. Data were as represented as mean \pm SEM and $P<0.05$ considered significant. ${ }^{*}$ was used for showing a significant difference between groups that are beside together (social $\times$ isolation and morphine-treated socialized (MTS) $\times$ morphine-treated isolated (MTI)).

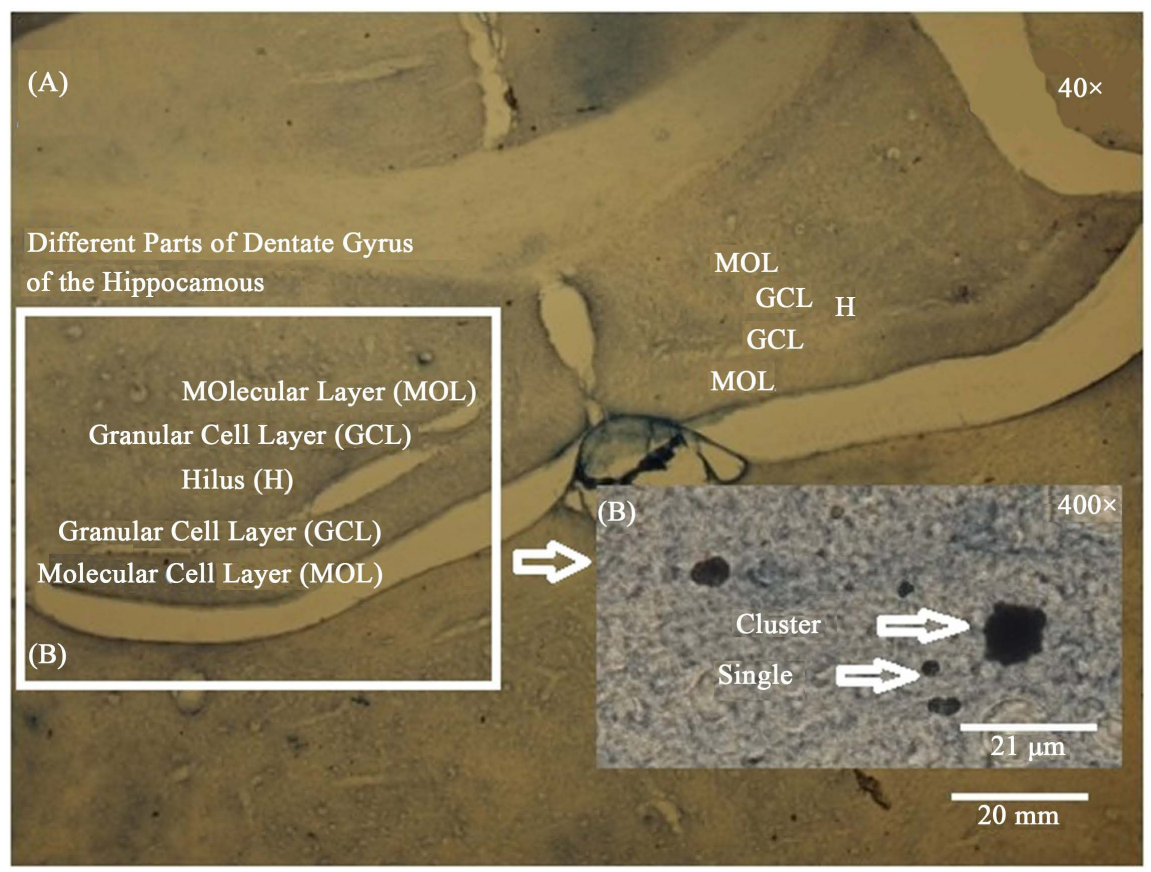

Figure 3. The above figure illustrates the right and left dentate gyrus of the hippocampus in $40 \times(\mathrm{A})$. The dentate gyrus (DG) of the hippocampus has three parts a) molecular layer b) hilus c) granular cell layer. The counting of BrdU-positive cells (neurons) has been done in different parts of DG. Right and left dentate gyruses (DG) have been counted. Inside (A), (B) shows BrdU-stained neurons that are recognizable in 400× in DG area that has been colored brown. They may be in single or cluster forms. 


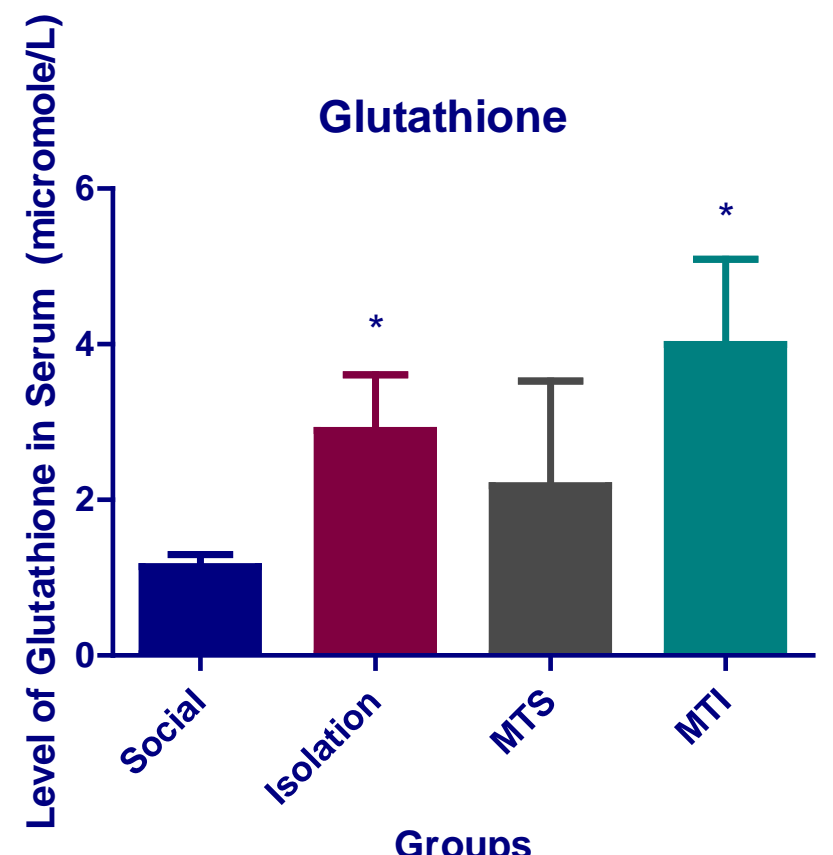

Figure 4. This figure illustrated serumic level of glutathione $(n=8)$. Data were as represented as mean \pm SEM and $P<0.05$ considered significant. ${ }^{*}$ was used for showing a significant difference between groups that are beside together (social $\times$ isolation and morphine-treated socialized $($ MTS $) \times$ morphine-treated isolated $($ MTI $)$ ).

Hippocampus as one of the components of reward circuit seems to have a critical role in modulation of reward-related behaviors. Neurogenesis as defined production of new neurons after birth in the dentate gyrus of the hippocampus in this regard has a regulatory function for adapting hippocampus functions to the ever-changing environment. Many studies support this idea that alternation of neurogenesis with changing environment is necessary for tolerance of stress-related stimuli [13].

Tail flick test mainly used to assess the sensitivity of the rats to reception of pain. It should be noted that Writhing test because of severe pain is not recommended in animal studies anymore [14]. Sensing pain involves two major components: environmental nociceptors and central modulation and perception. Rewarding nature of pain involves central part of pain perception and interpretation. So neurogenesis seems to be important for central pain modulators. Aversive to pain stimuli mainly is encoded by brain reward/motivational mesocorticolimbic circuitry [15].

Neurogenesis in the hippocampus has recently been paid much attention as a phenomenon that is motivated and inhibited in response to stress [16]. From this view reward and punishment can be assigned to regulate neurogenesis. Reduction and increased rate of neurogenesis is associated with keeping up with the relevant stress. Pain as an unwanted disturbance can be considered as a threat to life and neurogenesis from this point of view can be attributed a function to control stress. Nevertheless in this study reduction of neurogenesis and increased sensitivity to pain in tail flick test seems to have a negative correlation 
with each other. If it is considered that increase in neurogenesis is associated with more relief from pain this study would not support this idea. Instead, reduction of neurogenesis is associated with a reduction of sensitivity to pain stimuli. Reduction in neurogenesis may be an adaptive response to pain stimuli to reduce the sensation of the brain. From this view reduction of set-points of pain centers in the brain is the resulted outcome.

On the other hand increase in neurogenesis is associated with more cognitive function performance [17]. Reduction of memory performance may be necessary for reduction sense of pain. Since good memory may be brought to mind more pain and reduction of memory may be washing out pain memories.

Pain in some forms presented itself as vague a phenomenon that is unresponsive to drugs and medication. Proposing a reward-regulated modulator of pain would help to control intractable pain in many situations. By conditioning, the pain with reward evokes stimuli pain can be controlled. So here it is proposed that modulation of pain with alternation of neurogenesis as one of the parts of reward circuit would propose a new mechanism for pain modulation. The proposed mechanism for this study for reduction of pain is this that reduced neurogenesis increases reward center sensitivity to pain stimuli, and thereby controlling of pain. It should be noted that one of the main questions in treating pain is this that how long it takes painful stimuli arise pain and how fast the controllers of pain in the brain would act to control pain. In this study, it seems that neurogenesis modulates pain perception in a short time.

Glutathione according to recent studies is the most important antioxidant defense for brain abnormalities [18]. The more metabolism in the brain results in more free radical production. This free radical should be neutralized in order to prevent damages to brain tissues. In an increased stress related condition the production of free radicals increases because stress hormones also increase. This led to more free radical production. These hormones insufficiencies are reduced corticosterone in serum, unaltered catecholamines, and low blood glucose. Proapoptotic factors also overexpress in the brain [19]. Glutathione elevation in isolation states of this experiment may project the importance of the antioxidant factor for defending the devastating effects of isolation. So, this research also is high lightening the importance of this antioxidant for brain functions such as controlling pain.

\section{Conclusion}

Pain sensitivity is inversely related to the neurogenesis in isolation state. Morphine treatment also is effective for reducing sensitivity to pain, but isolation alone is more effective. Thus morphine and isolation are two important factors for reducing the sense of pain and reduction of neurogenesis also is an important factor in this regard.

\section{References}

[1] Kim, J., Ham, S., Hong, H., Moon, C. and Im, H.I. (2016) Brain Reward Circuits in 
Morphine Addiction. Molecules and Cells, 39, 645.

https://doi.org/10.14348/molcells.2016.0137

[2] Lledo, P.M., Alonso, M. and Grubb, M.S. (2006) Adult Neurogenesis and Functional Plasticity in Neuronal Circuits. Nature Reviews Neuroscience, 7, 179-193. https://doi.org/10.1038/nrn1867

[3] Ming, G.L. and Song, H. (2011) Adult Neurogenesis in the Mammalian Brain: Significant Answers and Significant Questions. Neuron, 70, 687-702. https://doi.org/10.1016/j.neuron.2011.05.001

[4] Vachon, P., Millecamps, M., Low, L., Thompsosn, S.J., Pailleux, F., Eaudry, F.B. Bushnell, C.M. and Stone, L.S. (2013) Alleviation of Chronic Neuropathic Pain by Environmental Enrichment in Mice Well after the Establishment of Chronic Pain. Behavioral and Brain Functions, 9, 1. https://doi.org/10.1186/1744-9081-9-22

[5] Han, X., Wang, W., Xue, X., Shao, F. and Li, N. (2011) Brief Social Isolation in Early Adolescence Affects Reversal Learning and Forebrain BDNF Expression in Adult Rats. Brain Research Bulletin, 86, 173-178. https://doi.org/10.1016/j.brainresbull.2011.07.008

[6] Beard, R.L. and O'Connor, M.K. (2015) Listening to Alzheimer's: The Role of Social Location. Psychosocial Studies of the Individual's Changing Perspectives in Alzheimer's Disease.

[7] Yorgason, J.T., Calipari, E.S., Ferris, M.J., Karkhanis, A.N., Fordahl, S.C., Weiner, J.L. and Jones, S.R. (2016) Social Isolation Rearing Increases Dopamine Uptake and Psychostimulant Potency in the Striatum. Neuropharmacology, 101, 471-479.

https://doi.org/10.1016/j.neuropharm.2015.10.025

[8] Peitz, G.W., Strickland, J.C., Pitts, E.G., Foley, M., Tonidandel, S. and Smith, M.A. (2013) Peer Influences on Drug Self-Administration: An Econometric Analysis in Socially Housed Rats. Behavioural Pharmacology, 24, 114-123. https://doi.org/10.1097/FBP.0b013e32835f1719

[9] Cecchi, M., Capriles, N., Watson, S. and Akil, H. (2008) Differential Responses to Morphine-Induced Analgesia in the Tail-Flick Test. Behavioral Brain Research, 194, 146-151. https://doi.org/10.1016/j.bbr.2008.06.034

[10] Chen, S.L., Ma, H.I., Han, J.M., Lu, R.B., Tao, P.L., Law, P.Y. and Loh, H.H. (2010) Antinociceptive Effects of Morphine and Naloxone in Mu-Opioid Receptor Knockout Mice Transfected with the MORS196A Gene. Journal of Biomedical Science, 17, 1. https://doi.org/10.1186/1423-0127-17-28

[11] Ibi, D., Takuma, K., Koike, H., Mizoguchi, H., Tsuritani, K., Kuwahara, Y., Kamei, H., Nagai, T., Yoneda, Y., Nabeshima, T. and Yamada, K. (2008) Social Isolation Rearing-Induced Impairment of the Hippocampal Neurogen Esis Is Associated with Deficits in Spatial Memory and Emotion-Related Behaviors in Juvenile Mice. Journal of Neurochemistry, 105, 921-932. https://doi.org/10.1111/j.1471-4159.2007.05207.x

[12] Dringen, R., Brandmann, M., Hohnholt, M.C and Blumrich, E.M. (2015) Glutathione-Dependent Detoxification Processes in Astrocytes. Neurochemical Research, 40, 2570-2582. https://doi.org/10.1007/s11064-014-1481-1

[13] Lucassen, P.J., Meerlo, P., Naylor, A.S., Van Dam, A.M., Dayer, A.G., Fuchs, E., Oomen, C.A. and Czeh, B. (2010) Regulation of Adult Neurogenesis by Stress, Sleep Disruption, Exercise and Inflammation: Implications for Depression and Antidepressant Action. European Neuropsychopharmacology, 20, 1-17. https://doi.org/10.1016/j.euroneuro.2009.08.003

[14] Gawade, S.P. (2012) Acetic Acid Induced Painful Endogenous Infliction in Writhing Test on Mice. Journal of Pharmacology \& Pharmacotherapeutics, 3, 348. 
https://doi.org/10.4103/0976-500X.103699

[15] Navratilova, E., Atcherley, C.W. and Porreca, F. (2015) Brain Circuits Encoding Reward from Pain Relief. Trends in Neurosciences, 38, 741-750.

https://doi.org/10.1016/j.tins.2015.09.003

[16] Czeh, B., Muller-Keuker, J.I., Rygula, R., Abumaria, N., Hiemke, C., Domenici, E. and Fuchs, E. (2007) Chronic Social Stress Inhibits Cell Proliferation in the Adult Medial Prefrontal Cortex: Hemispheric Asymmetry and Reversal by Fluoxetine Treatment. Neuropsychopharmacology, 32, 1490-503.

https://doi.org/10.1038/sj.npp.1301275

[17] Ferragud, A., Haro, A., Sylvain, A., Velazquez-Sanchez, C., Hernandez-Rabaza, V. and Canales, J.J. (2010) Enhanced Habit-Based Learning and Decreased Neurogenesis in the Adult Hippocampus in a Murine Model of Chronic Social Stress. Behavioral Brain Research, 210, 134-139. https://doi.org/10.1016/j.bbr.2010.02.013

[18] Bains, V.K. and Bains, R. (2015) The Antioxidant Master Glutathione and Periodontal Health. Dental Research Journal, 12, 389-405.

https://doi.org/10.4103/1735-3327.166169

[19] Djordjevic, A., Adzic, M., Djordjevic, J. and Radojcic, M.B. (2009) Chronic Social Isolation Is Related to Both Upregulation of Plasticity Genes and Initiation of Proapoptotic Signaling in Wistar Rat Hippocampus. Journal of Neural Transmission, 116, 1579-1589. https://doi.org/10.1007/s00702-009-0286-x

\section{Scientific Research Publishing}

\section{Submit or recommend next manuscript to SCIRP and we will provide best service for you:}

Accepting pre-submission inquiries through Email, Facebook, LinkedIn, Twitter, etc. A wide selection of journals (inclusive of 9 subjects, more than 200 journals) Providing 24-hour high-quality service User-friendly online submission system Fair and swift peer-review system Efficient typesetting and proofreading procedure Display of the result of downloads and visits, as well as the number of cited articles Maximum dissemination of your research work

Submit your manuscript at: http://papersubmission.scirp.org/ Or contact wjns@scirp.org 